\section{Passenger mutations take the wheel}

\section{By Tim Fulmer, Senior Writer}

Two research groups have independently shown that targeting genes carrying passenger mutations blocked growth of cancer cells and improved survival in mouse models. ${ }^{1,2}$ Although precise therapeutic windows for such a strategy remain to be determined, the researchers are already planning to develop therapeutics that exploit the presence of those mutations to inhibit tumor growth.

Hotly pursued cancer targets are usually proteins encoded by tumor suppressor genes and oncogenes such as BRAF and BCR-ABL tyrosine kinase because mutations in those genes promote malignant transformation and drive disease progression. However, most solid tumors also accumulate thousands of mutations in other genes over the course of disease.

Those passenger mutations may not promote tumor development like driver mutations but can nonetheless disrupt genes in metabolic and housekeeping pathways that are necessary for long-term tumor survival. ${ }^{3-6}$ Thus, targeting the proteins encoded by those mutated genes could offer a way to slow tumor growth or at least sensitize tumor cells to cancer therapies that hit other pathways.

The challenge is identifying candidate genes susceptible to such passenger mutations in tumors. Two academic groups approached the problem using detailed genetic analyses of the solid tumor genome.

A team led by Ronald DePinho, president of The University of Texas MD Anderson Cancer Center, primarily focused on passenger mutations that occur in housekeeping genes that are near a particular tumor suppressor region in the tumor genome. The other study, led by William Hahn and Rameen Beroukhim, professors of medicine at the Dana-Farber Cancer Institute, focused on passenger mutations that occur in housekeeping genes across many regions of the tumor genome. Hahn is also associate professor of medicine and Beroukhim is also assistant professor of medicine at Harvard Medical School.

DePinho and colleagues initially noted that many metabolic and housekeeping genes occur as redundant homologs to ensure cells remain viable if one gene homolog is deleted or disrupted. They hypothesized that if a passenger mutation removed or inactivated one homolog of a housekeeping gene, the tumor cells would become highly sensitive to inhibition of the only remaining homolog.

To find such redundant housekeeping genes, the researchers looked at The Cancer Genome Atlas data set for glioblastoma to find deletions in genes involved in essential cell activities.?

One of their top hits was a gene encoding the essential glycolytic enzyme enolase $1 \alpha$ (ENO1), which is located next to a tumor suppressor locus that is deleted in $1 \%-5 \%$ of glioblastomas. That suggested the

Table 1. Potentially targetable passenger mutations in cancer. Two papers, one published in Nature and the other in Cell, identified mutations that occur in genes involved in the cellular housekeeping and metabolic functions of tumor cells. Below are the top hits.

\begin{tabular}{|c|c|c|}
\hline \multicolumn{3}{|c|}{ Muller et al. } \\
\hline Gene abbreviation & Encoded protein & Cellular pathway \\
\hline ENO1 & Enolase $1 \alpha$ & Glycolysis and gluconeogenesis \\
\hline$H 6 P D$ & Hexose-6-phosphate dehydrogenase & Pentoase phosphate shunt \\
\hline KIF1B & Kinesin family member $1 \mathrm{~B}$ & Chromosomal segregation \\
\hline NMNAT1 & Nicotinamide nucleotide adenylyltransferase 1 & Nicotinamide dinucleotide (NAD) biosynthesis \\
\hline$U B E 4 B$ & Ubiquitination factor E4B & Ubiquitin-dependent degradation \\
\hline ACO1 (IRP1) & Aconitase 1 & Iron metabolism and citric acid cycle \\
\hline KLHL9 & Kelch-like 9 & Chromosomal segregation \\
\hline PANK1 & Pantothenate kinase 1 & Acetyl-CoA biosynthesis \\
\hline KIF20B & Kinesin family member $20 \mathrm{~B}$ & Chromosomal segregation \\
\hline \multicolumn{3}{|c|}{ Nijhawan et al. } \\
\hline Gene abbreviation & Encoded protein & Cellular pathway \\
\hline PSMC2 & Proteasome $26 \mathrm{~S}$ subunit ATPase 2 & Proteasome-mediated degradation \\
\hline EIF2B2 & Eukaryotic translation initiation factor $2 \mathrm{~B}$ subunit $2 \beta$ & Protein synthesis \\
\hline EEF2 & Eukaryotic translation elongation factor 2 & Protein synthesis \\
\hline PHF5A & PHD finger protein $5 \mathrm{~A}$ & Spliceosome-mediated mRNA splicing \\
\hline HPGD (15-PGHD) & Hydroxyprostaglandin dehydrogenase 15 NAD & Prostaglandin metabolism \\
\hline RPS15 & Ribosomal protein S15 & Component of 40 S ribosome subunit \\
\hline SNRPB & Small nuclear ribonucleoprotein polypeptides B and B1 & Pre-mRNA splicing \\
\hline POLR2F & Polymerase RNA II DNA-directed polypeptide F & Component of RNA polymerase \\
\hline USPL1 & Ubiquitin-specific peptidase-like 1 & Ubiquitin-dependent degradation \\
\hline
\end{tabular}


ENO1 gene might also be susceptible to deletion or inactivation. Indeed, expression profiling showed that 5 of 359 glioblastoma samples and 2 glioblastoma cell lines lacked expression of ENO1 when the tumor suppressor locus also was missing.

As expected, those cell lines were highly sensitive to inhibition of enolase $2 \gamma$ neuronal (ENO2), the remaining enolase homolog in neuronal cells. In two glioblastoma cell lines lacking ENO1, small hairpin RNA against ENO2 inhibited cell growth.

In the same cell lines, the small molecule enolase inhibitor phonoacetohydroxamate (PHAH) lowered growth compared with vehicle. In wild-type glioblastoma cells expressing both ENO1 and ENO2, the inhibitor failed to reduce growth.

The final result confirmed that a passenger deletion in one homolog was sufficient to make tumor cells susceptible to loss or inhibition of the remaining homolog.

The findings, which were published in Nature, are part of a research program that began at Dana-Farber and then continued at MD Anderson when DePinho was appointed president of the center last year.

In the second paper, Hahn, Rameen and colleagues set out with the similar hypothesis that loss of tumor suppressor genes might involve loss of other nearby genes involved in the stress response, which could reduce the fitness of tumor cells.

To test that idea, the researchers undertook a systematic investigation of the copy number status of more than 7,000 genes in 86 different cancer cell lines. ${ }^{8}$ The group narrowed the list to 56 candidate genes for which expression of only a single copy increased susceptibility to cell death compared with expression of both copies of the gene.

Many of the candidate genes encoded proteins that were subunits of protein complexes essential to cellular survival, including the spliceosome, proteasome and the ribosome. The top hit was the gene encoding proteasome 26S subunit ATPase 2 (PSMC2), which is part of

"It might be useful to develop specific catalytic inhibitors of housekeeping genes, then partner with informatics/ genomics providers to establish in which tumors those inhibitors might be particularly valuable."

-Erica Golemis,

Fox Chase Cancer Center the regulatory complex of the $26 \mathrm{~S}$ proteasome.

To test the therapeutic potential of suppressing PSMC2 in vivo, the researchers used tumor-specific nanoparticles to deliver anti-PSMC2 small interfering RNA to mouse ovarian xenografts. In xenografts expressing only a single copy of PSMC2, the siRNA decreased tumor burden by more than $75 \%$ and increased

survival compared with control siRNA.

However, in xenografts expressing normal levels of PSMC2, the siRNA failed to reduce tumor burden or improve survival, confirming that a passenger deletion in one copy of PSMC2 made ovarian cancer cells more susceptible to inhibition of the second copy.

The results were published in Cell.

Passenger mutations "may be the Achilles heel of cancer genomes" and "should create many opportunities for personalized medicine," wrote Ben Lehner and Solip Park in a commentary accompanying the Nature paper. ${ }^{9}$ Lehner and Park are researchers in the Centre for Genomic Regulation at Pompeu Fabra University.

\section{From mutations to molecules}

Both groups now plan to develop inhibitors of the proteins identified in the papers. The teams also will look for passenger mutations in other proteins and tumor types.

The enolase inhibitor used in the Nature paper "needs further exploration in terms of off-target pharmacology, pharmacokinetics and safety profile before it could be considered for clinical development," lead author Florian Muller told SciBX.

He noted that enolase is a relatively unexplored target, and as a result the team is "performing high throughput screens to look for alternative chemical scaffolds that may be better starting points" for drug development.

Based on the findings in the Cell paper, Hahn said the Dana-Farber group plans to identify ways to inhibit specific proteasome subunits such as PSMC2. He said the researchers also want to expand their approach to other tumor types to identify additional candidate genes but declined to provide additional details.

DePinho, Muller and colleagues will continue to look at glioblastoma. "There are many more ENO1/ENO2-type pairs in the glioblastoma cancer genome, and we would like to experimentally validate other examples as we did in the Nature paper for ENO1," said Muller, who is an instructor at MD Anderson.

Other researchers on the DePinho team include Simona Colla, an instructor at MD Anderson, and Elisa Aquilanti, a medical student at the Albert Einstein College of Medicine of Yeshiva University.

\section{Targeting the housekeeper}

The two papers provide a rationale for tumor-selective targeting of metabolic and housekeeping genes, which have generally not been considered promising targets in oncology (see Table 1, "Potentially targetable passenger mutations in cancer").

Housekeeping genes have been avoided as drug targets "because of the rational assumption by the drug discovery community that it would be difficult to develop a therapeutic window when inhibiting a protein that every cell in the body needs," said Erica Golemis.

She said the Nature paper now points out that "this limitation does not necessarily apply and hence opens up a much broader class of protein targets for therapeutic development. It might be useful to develop specific catalytic inhibitors of housekeeping genes, then partner with informatics/genomics providers to establish in which tumors those inhibitors might be particularly valuable," she said.

Golemis, deputy CSO and VP of the Fox Chase Cancer Center, is using RNAi screens to identify proteins that influence the sensitivity of cancer cells to epidermal growth factor receptor (EGFR)-based therapies. ${ }^{10}$

Marion Dorsch, VP of biology at Agios Pharmaceuticals Inc., said the two papers "underscore the need for sophisticated genetics and metabolomics capabilities to identify the right intervention points in these pathways where cancer cells are unable to compensate but normal cells can."

Agios mines genomic data and conducts large-scale metabolomics experiments in tumor tissues to identify sensitive metabolic nodes in tumors that are vulnerable to inhibition. The company's lead program is in preclinical development and focuses on the cancer metabolism target isocitrate dehydrogenase 1 (IDH1).

Agios also has a discovery-stage program focused on another cancer metabolism target, pyruvate kinase M2 isozyme (PKM2). In 2009, Agios 


\section{ANALYSIS}

\section{TARGETS \& MECHANISMS}

researchers published in Nature that mutations in IDH1 lead to excess levels of the cancer-associated metabolite 2-hydroxyglutarate. ${ }^{11}$

\section{Narrow window}

The genome-based approaches used in the two papers could have limitations, including a narrow therapeutic window and the potential to engender treatment resistance.

"First, this approach requires expensive, detailed genotyping of many malignancies to find the few for which each specialized genespecific agent might be applicable," said Paul Bingham, VP of research at Cornerstone Pharmaceuticals Inc. "Second, it remains to be seen how many common tumors will offer frequently deleted redundant and/or essential genes. Finally, given that metabolism is complex in its pathway branching, the approach could be susceptible to developing resistance."

Cornerstone's strategy is to attack tumor-specific regulatory processes that are general and not confined to tumors that have genespecific alterations. The approach ensures that "we get broad potential tumor coverage and off-target adverse effects on noncancer cells are mitigated if not eliminated," said Bingham.

Cornerstone's lead compound is CPI-613, a lipoate analog that targets the metabolic enzyme pyruvate dehydrogenase. The compound is in Phase I/II testing to treat advanced hematological malignancies. In mouse xenograft models of non-small cell lung cancer (NSCLC) and pancreatic cancer, CPI-613 decreased tumor volume and increased survival compared with vehicle. ${ }^{12}$

Ultimately, the major challenge for targeting housekeeping genes in cancer "is to demonstrate a therapeutic window and examine toxicity," said Jason Moffat. "We know these housekeeping genes are essential for the proliferation of most cell types, so identifying the cohort of patients that demonstrate sensitivity to a possible drug is going to be key moving forward." Moffat is assistant professor of molecular genetics at the University of Toronto.

Moffat said his lab is developing profiles of essential genes for multiple cancer cell lines and then combining that information with genomic information to develop a genetic interaction atlas of cancer cells.
The findings in both papers are covered by patents and available for licensing.

Fulmer, T. SciBX 5(35); doi:10.1038/scibx.2012.915

Published online Sept. 6, 2012

\section{REFERENCES}

1. Muller, F.L. et al. Nature; published online Aug. 15, 2012; doi:10.1038/nature11331

Contact: Ronald A. DePinho, University of Texas MD Anderson Cancer Center, Houston, Texas e-mail: rdepinho@mdanderson.org

2. Nijhawan, D. et al. Cell; published online Aug. 17, 2012; doi:10.1016/j.cell.2012.07.023

Contact: William C. Hahn, Harvard Medical School, Boston, Mass.

e-mail: william_hahn@dfci.harvard.edu

Contact: Rameen Beroukhim, same affiliation as above

e-mail: rameen_beroukhim@dfci.harvard.edu

3. Greenman, C. et al. Nature 446, 153-158 (2007)

4. Stratton, M.R. et al. Nature 458, 719-724 (2009)

5. Sjöblom, T. et al. Science 314, 268-274 (2006)

6. Fröhling, S. et al. Cancer Cell 12, 501-513 (2007)

7. The Cancer Genome Atlas Research Network. Nature 455, 1061$1068(2008)$

8. Cheung, H.W. et al. Proc. Natl. Acad. Sci. USA 108, 12372-12377 (2011)

9. Lehner, B. \& Park, S. Nature 488, 284-285 (2012)

10. Astsaturov, I. et al. Sci. Signal. 3, ra67 (2010)

11. Dang, L. et al. Nature 462, 739-744 (2009)

12. Zachar, Z. et al. J. Mol. Med. (Berl.) 89, 1137-1148 (2011)

COMPANIES AND INSTITUTIONS MENTIONED

Agios Pharmaceuticals Inc., Cambridge, Mass. Albert Einstein College of Medicine of Yeshiva University, New York, N.Y.

Cornerstone Pharmaceuticals Inc., Cranbury, N.J.

Dana-Farber Cancer Institute, Boston, Mass.

Fox Chase Cancer Center, Philadelphia, Pa.

Harvard Medical School, Boston, Mass.

Pompeu Fabra University, Barcelona, Spain

The University of Texas MD Anderson Cancer Center, Houston, Texas

University of Toronto, Toronto, Ontario, Canada 